\title{
RiboTagger: fast and unbiased 16S/18S profiling using whole community shotgun metagenomic or metatranscriptome
}

\section{surveys}

\author{
Chao Xie ${ }^{1,5^{*}}$, Chin Lui Wesley Goi ${ }^{1}$, Daniel H. Huson ${ }^{2,3}$, Peter F. R. Little ${ }^{1,3,4}$ and Rohan B. H. Williams ${ }^{1}$
}

From 15th International Conference On Bioinformatics (INCOB 2016)

Queenstown, Singapore. 21-23 September 2016

\begin{abstract}
Background: Taxonomic profiling of microbial communities is often performed using small subunit ribosomal RNA (SSU) amplicon sequencing (16S or 18S), while environmental shotgun sequencing is often focused on functional analysis. Large shotgun datasets contain a significant number of SSU sequences and these can be exploited to perform an unbiased SSU-based taxonomic analysis.

Results: Here we present a new program called RiboTagger that identifies and extracts taxonomically informative ribotags located in a specified variable region of the SSU gene in a high-throughput fashion.

Conclusions: RiboTagger permits fast recovery of SSU-RNA sequences from shotgun nucleic acid surveys of complex microbial communities. The program targets all three domains of life, exhibits high sensitivity and specificity and is substantially faster than comparable programs.
\end{abstract}

Keywords: Microbial ecology, Short subunit ribosomal RNA, Microbial community profiling, Sequence analysis

\section{Background}

Studying the composition and dynamics of microbial communities is a key problem in microbiome research and microbial ecology [1]. Traditionally, these studies have been based on isolating and sequencing short subunits of the $16 \mathrm{~S}$ and $18 \mathrm{~S}$ genes, present in bacteria or archea, and eukaryotes, respectively. Typically most studies now make use of amplicon sequencing to obtain such data from complex microbial communities [2, 3]. The PCR amplicon technique was very useful when sequencing power was limited, however, with the increasing power and complexity of the new generation of sequencing technologies, the broad advantages of amplicon sequencing are starting to be balanced by major limitations, which include PCR primer

\footnotetext{
* Correspondence: xiechaos@gmail.com

${ }^{1}$ Singapore Centre for Environmental Life Sciences Engineering, National University of Singapore, Singapore 117456, Singapore

${ }^{5}$ Current address: Human Longevity Inc, Singapore, Singapore

Full list of author information is available at the end of the article
}

selection and amplification bias [4, 5]. In particular, no PCR primers are able to amplify all known bacterial taxonomic groups efficiently and uniformly [5], which leads to biased rRNA profiling analysis, and the use of short read technologies, notably Illumina, results in a complex, interdependent chain of technical decisions, that can heavily influence the subsequent community profiling results [6].

Within microbiome research, there is increasing use of whole community gDNA surveys (i.e. shotgun metagenomics), which offer, at theoretically, a less biased view of community composition than using from amplicon based methods, by eliminating dependency on $16 \mathrm{~S}$ primers [7]. In practice however, the interpretation of shotgun metagenome data is heavily dependent on having access to reference genomes of community members, without which substantial limitations of interpretation may arise [8]. While the intended use of these shotgun data is typically to capture functional capacity of a community [9], or to permit member genome recovery [8], it has been recognized 
that whole commnuity shotgun surveys will of course contain a substantial number of reads derived from SSUrRNA genes, and these can be exploited for the purposes of community profiling [10-17]. Similarly, when using total RNA metatranscriptome sequencing, rRNA often account for $95 \%$ of reads sequenced, and thus provides coverage of SSU diversity to great depth. Within this general area, a number of examples of this approach have been undertaken and several software implementations of this approach are now available $[13,16,17]$, mostly based on the use of Hidden Markov Models to capture reads of SSU-origin from the total read population [10-14, 16, 17].

Here we present a new open source software package, RiboTagger, (https://github.com/xiechaos/ribotagger) to analyze rRNA data from shotgun sequencing reads. The software takes raw metagenome or metatranscriptome sequencing reads in FASTQ or FASTA files as input, and is able to process billions of Illumina HiSeq reads under an hour. RiboTagger produces a BIOM formatted files for downstream analysis in standard packages like QIIME [18] or MEGAN [19]. It is equally sensitive over all known bacterial and archaeal phyla and classes, and highly specific in not classifying non-rRNA sequences as rRNA.

\section{Implementation}

We start with genomic DNA or total RNA from a microbial community that has been sequenced, then attempt to recover sequencing reads covering a particular region on $16 \mathrm{~S}$ rRNA gene using a short conserved recognition sequence (RS). A short sequence adjacent to the RS is used as the tag sequence (TS) to represent the origin of the $16 \mathrm{~S}$ gene. For this strategy to be feasible with short-read sequencing technologies, by nature both RS and TS have to be short but informative and in particularly the RS must be conserved across all taxonomic branches, while the TS must be diverse with high taxonomic resolution. The boundaries of each hypervariable region are logical candidates for being RS, which we call ribotags from here on. After examining all bacterial and archaeal $16 \mathrm{~S}$ sequences in the RDP database [20] we designed a combination of probe patterns and Position Specific Scoring Matrices (PSSM) to recognize the conserved site immediately outside a hypervariable region as RS, which we describe as a universal recognition profile (Fig. 1). The short region in the hypervariable region adjacent to the $\mathrm{RS}$ is then considered as a candidate tag sequence for the hypervariable region (Fig. 1). In this PSSM-based detection, the RS sequences are 23 n.t. in length and the TS are typically 33 n.t. in length. The lengths of both RS and TS were empirically determined so as to achieve a good trade-off between sensitivity and specificity, while maintaining a total length that is smaller than a typical sequencing read. For each of the three domains of life and each of the most commonly used variable regions, namely V4, V5, V6 and V7, we computed a universal recognition profile by analyzing the Greengenes database [21] (Fig. 2). To improve sensitivity, our implementation provides and can utilize a set of 17-30 supplementary taxon-specific recognition profiles for each of the variable regions.

\section{Results and discussion \\ Validation analyses}

Application of our universal profiles to the Greengenes database gave rise to a set of 90,061 ribotags. Each ribotag was assigned a taxon based on the set of SSU sequences that contain it, using the majority taxon in the case of discordance. The sensitivity of each of the profiles is over $95 \%$ percent (Fig. 2), as established by applying the profiles to all SSU sequences in the SILVA database [22]. To test the false positive rate, we ran the profiles on 5.6 billion faux reads, obtained by sampling all $80 \mathrm{nt}$ non-overlapping fragments in the RefSeq database (release 66), calculating the false positive rate to be at most $2 \times 10^{-6}$ in all cases (Fig. 2). To address the problem of the extent to which sequencingerror can generate false ribotags, RiboTagger estimates the expected frequency of each ribotag due to sequencing error,

\section{(1)}

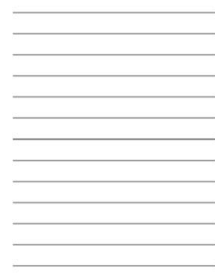

Shotgun Reads

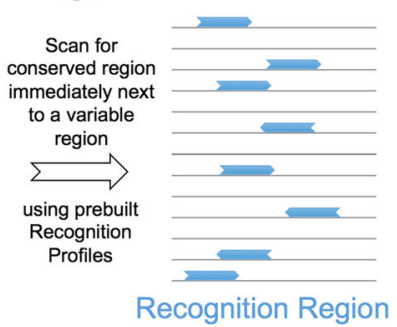

(2)

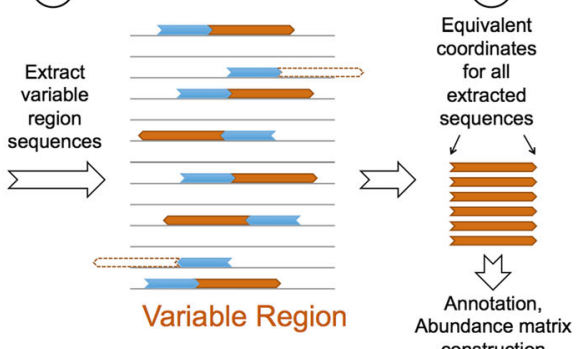

Fig. 1 Schematic representation of RiboTagger detection scheme. Starting with shotgun sequencing reads from (either gDNA or cDNA) RiboTagger procedes as follows; (1) all reads are screened using PSSMs for the presence of a conserved recognition sequence (blue arrowed rectangles) adjacent to $\mathrm{V}$-regions using a cohort of pre-defined recognition profiles; (2) for reads that are positive for recognition sequences, the adjacent V-region tag sequence is extracted, assuming if sufficient length is available (dashed blue rectangles denote in sufficient length in the tag sequence); and (3) for related tag sequences, equivalent coordinates are defined, prior to counting and annotation 


\begin{tabular}{|c|c|c|c|c|}
\hline & & Universal Recognition Profile & Sensitivity & $\begin{array}{c}\text { False } \\
\text { Positive } \\
\text { Rate }\end{array}$ \\
\hline \multirow{3}{*}{ V4 } & B & TGGCeGTAAAGGG CGIAGGCG & $96.9 \%$ & 3.4E-07 \\
\hline & A & 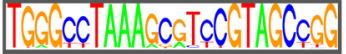 & $96.2 \%$ & $1.7 \mathrm{E}-10$ \\
\hline & $\mathrm{E}$ & "CGAGTIAAAACCTCGTAGTTGAA & $96.9 \%$ & $9.6 \mathrm{E}-07$ \\
\hline \multirow{3}{*}{ V5 } & B & GTAGTCCACGCGGTAAACGATG & $96.6 \%$ & $1.2 \mathrm{E}-06$ \\
\hline & A & GTAGTCG AGCTGTAAAC & $95.6 \%$ & 3.3E-08 \\
\hline & $\mathrm{E}$ & 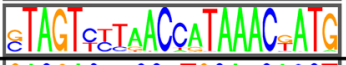 & $96.5 \%$ & $5.1 \mathrm{E}-07$ \\
\hline \multirow{3}{*}{ V6 } & B & GACGACACCCATGCAECACCT & $98.3 \%$ & $1.9 \mathrm{E}-07$ \\
\hline & A & & $95.6 \%$ & $3.1 \mathrm{E}-08$ \\
\hline & $\mathrm{E}$ & AAGAACGCCCATCCACCACCA & $97.7 \%$ & $6.0 \mathrm{E}-07$ \\
\hline \multirow{3}{*}{ V7 } & B & AAGTCCCGCACGACECAACCC & $98.4 \%$ & $2.0 \mathrm{E}-06$ \\
\hline & A & AAGTCAGGCAACGACCGCACCC & $95.3 \%$ & $1.6 \mathrm{E}-08$ \\
\hline & E & AATTCCG TAACGACGAGACcD & $97.2 \%$ & 4.1E-07 \\
\hline
\end{tabular}

Fig. 2 Universal recognition profiles for each of the variable regions V4V7 used to target bacteria (B), archaea (A) and eukaryotes (E), respectively, with corresponding observed sensitivities and false positive rates

based on the observed frequencies of ribotags that differ by one letter and a simple probabilistic model.

To determine how well ribotags can differentiate between organisms at a given taxonomic rank, we computed the concordance of all ribotags obtained from an analysis of the Greengenes database. We consider a ribotag as fully concordant (or to have concordance 1) at a given taxonomic rank, if all database sequences that contain that ribotag have the same taxonomic assignment at the given rank. For example, the concordance is between 0.95 and 1.00 if between 95 and $100 \%$ of the database sequences that contain the ribotag all have the same taxon assignment. The percentages of V4 ribotags with a given range of concordance (i.e. $1,[0.95,1),[0.95,0.90)$, etc.) for different taxonomic ranks as annotated in the Greengenes database are shown in Fig. 3a. Approximately $80 \%$ of all ribotags are fully concordant on the species level, while practically all reads are concordant at the phylum level. Results are similar for V6 ribotags, while the number of fully concordant ribotags is about $5 \%$ lower for V5 and V7 (data not shown). We also examined the degree of concordance against de novo OTU clusters defined by Greengenes over a range of percent identity thresholds, showing that for ribotags in clusters defined at $99 \%$ similarity, over $80 \%$ show no sequence differences (Fig. 3b).

Due to the short length of the $16 \mathrm{~S}$ tags, we need to consider the possible influence of sequencing errors explicitly. PCR artifacts during library generation would lead to abnormally high numbers of reads with identical starting position and sequence content. Therefore, simply counting

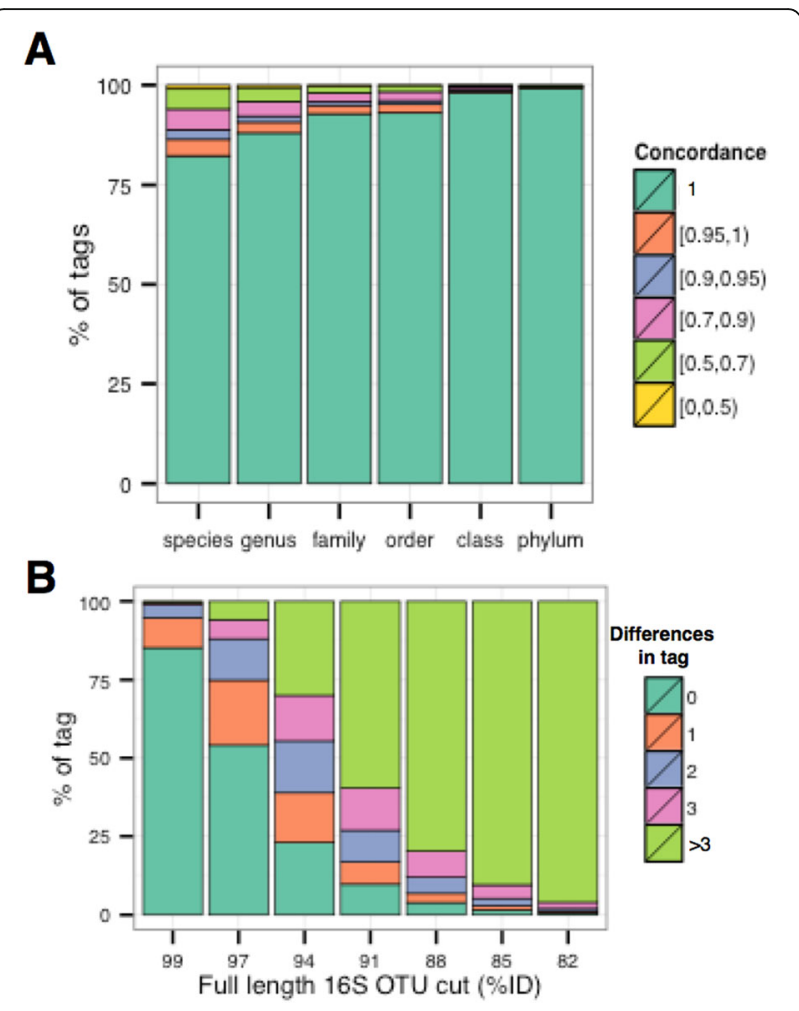

Fig. 3 Percentage of V4 ribotags with a given range of concordance for different taxonomic ranks as ( $\boldsymbol{a}$ ) annotated in the Greengenes database and (b) as measured against Greengenes de novo OTU clusters as a function of cluster formation threshold

sequencing reads with different starting position can detect possible PCR artifacts. Using stringent quality value filtering for each nucleotide that covers $16 \mathrm{~S}$ tags can also the impact of limit potential sequencing errors, which results extremely low error probability for each sequencing read considered. For example, with quality value threshold 30 , the probability of sequencing error is only $0.1 \%$. In addition, we also calculate the number of expected number of occurrence of any $16 \mathrm{~S}$ tag, assuming the tag is not present at all. Assuming the tag of interest is not present, given the observed average sequencing error probability at each nucleotide position along the tag and the abundance of all other tag sequences with one nucleotide difference in the data, we can calculate the expected abundance of the tag of interest by sequencing error. Firstly, for the tag under study, all tag sequences with one nucleotide difference from the tag of interest are collected. Each of the $1 \mathrm{nt}$ neighbors will contribute $E=n \times e / 3$ false positive counts to the tag of interest, where $n$ is the number of reads covering the $1 \mathrm{nt}$ neighbour and $e$ is the observed error probability for the difference position on the tag of interest. The sum of $E$ for all $1 \mathrm{nt}$ neighbours gives the expected false positive tag of interest due to one sequencing error. Multiple sequencing errors can be also considered in a similar fashion but due to the quality-value filtering step, we can consider the 
probability of observing multiple sequencing errors in ribotag sequences as negligible.

\section{Example}

To illustrate the results obtained from RiboTagger, we ran RiboTagger against a coupled DNA-Seq and RNA-Seq dataset generated from an activated sludge community of an operational wastewater treatment (Ulu Pandan Water Reclamation Plant, Northworks, Tank 3C), operated by the Public Utilities Board, Republic of Singapore; samples obtained between 10/08/2012 and 17/08/2012). Genomic DNA and total RNA sequencing were each performed on a HiSeq2500 Rapid run using 250 bp paired end sequencing for DNA samples, and 150 bp paired end read sequencing for RNA samples. We observed a total of 4686 V4-region ribotags in the entire dataset. From the DNA dataset, we can estimate whether the number of observed $16 \mathrm{~S}$ tags is as expected using the following rough approximation: if we assume there are up to 5000 genes in a typical free-living bacteria [23], and if we neglect the likely variation in $16 \mathrm{~S}$ copy number across the member species in the community, and assume that one of those is a $16 \mathrm{~S}$ gene with roughly 9 equally sized V-regions and 10 equally sized conserved regions, then we would expect approximately 308,505,950/ $(19 \times 5000)=3247$ reads to originate from a single V-region in our analysis, which is a conservative underestimate to our observed number. For the RNA data, we report only results for one sample to for purposes of illustration, specifically with a total of 41,523,808 RNA reads available after QC filtering., we obtained a total of 4867 tags, of which the top 11 accounted for $20 \%, 80 \%$ and $95 \%$ of community membership (as described by total number of reads). Collectively there are 327 unique genera detected, 203 families, 128 orders and 51 phyla, The majority of annotated tags at kingdom level (1450) were attributable to bacteria, as expected in this community, with 34 and 40 tags being assigned to archaea and eukaryota, respectively. Approximately 3343 tags could not be assigned any annotation using SILVA v119 [22], including 1 and 41 in the top 20\% and 50\% of the community, respectively. These latter results highlight the substantial numbers of unknown taxa residing in complex microbial communities.

\section{Comparison to related programs}

Using the RNA dataset described above, we compared the computational time of RiboTagger against two other existing search tools, RiboFrame [16] and SSUSearch [17], which are designed for retrieval of $16 \mathrm{~S}$ sequences from whole metagenomic sequencing datasets. The comparison was carried out on a standalone server with the following specifications-2 Intel Xeon X7542 (18 M Cache, $2.66 \mathrm{GHz}$ ) CPUs, $128 \mathrm{~GB}$ memory, internal $146 \mathrm{~GB}$ HDD and external 8 TB RAID HDD. RiboTagger took approximately 90 min to complete, and we observed RiboTagger to be approximately 6 times faster than RiboFrame $(\sim 9 \mathrm{~h})$ and 6.6 times faster than SSUSearch $(\sim 10 \mathrm{~h})$. As RiboTagger and SSUsearch both used SILVA annotations, we compared the identity and relative abundance of detected phlya between both programs. In total 112 phyla were detected (51 with Ribotagger and 86 with SSUsearch) of which 25 were common between the two methods. The number of unclassifiable sequences differed, with $21.6 \%$ being called in SSUsearch and $47.5 \%$ in RiboTagger, however, of the 25 common phyla, 7 accounted for 95\% of community composition in each set of results. The overall correlation between relative abundance was 0.99 (with 25 common phyla) and 0.88 (using all 112 phyla with non-detects set as zero). We note that while some of these differences may be accounted for by different database versions (SILVA v115 and SILVA v119 for SSUearch and Ribotagger, respectively) and/or handling of unclassifible sequences, these results suggest that further investigation of the differences between detection methodologies are warranted.

\section{Practical aspects}

RiboTagger is a implemented as a platform independent Perl program. It can be executed in a single command in several modes with are briefly described here, along with their respective outputs. Full details can be found on the RiboTagger project page on Github (see Availability and Requirements)

\section{A. Single input file mode}

The most basic way to run RiboTagger is using a single FASTQ file (including with either gzip and bzip2 compression), which will generate an output file formatted as a table with the following fields:

- tag: the tag sequence for the variable region

- $\mathrm{n}$ : the number of reads that contains this tag

- npos: the number of different locations of the tag on their source reads (a large value of $n$ and a small value of npos indicates the presence of duplicated reads or would be observed if applied to amplicon sequencing reads).

- fp: the number of reads you would expect to see this tag due to sequencing errors alone

- long.total.count: the number of reads containing a longer sequence of this tag (see the-long option)

- long1.count, long2.count, long3.count: number of reads containing the most abundant variants of this tag's long sequences (low long1.count/ long.total.count ratio indicates that this tag is very likely representing a mixture of "species")

- long1, long2: the most abundant long representive sequences of this tag

\section{B. Multiple input file mode}

For multiple input files, RiboTagger can return a series of files. Data from paired end read data can also be 
combined into a single output. The.tab file returns a table of unnormalized read counts, with ribotags indexed in rows and samples indexed in columns. The.anno files contains QC, metadata and annotations, if available, for the same set of ribotags with the following columns fields:

- tag: the ribotag sequence

- use: "tag" or "long", whether the annotation was based on the short tag or long representative sequence

- taxon_level: taxa rank of this annotation of this tag

- taxon_data: taxa rank of the most specific annotation appeared in the database (SILVA or Greengenes) for this tag

- long: the long representative sequence of this tag

- long_total: the number of samples having any long representative sequence

- long_this: the number of samples having this long sequence as its major representative of this tag

- support: the number of database sequences having this tag or long sequence

- confidence: the proportion of the database sequences agreed on this annotation

- k, p, c, o, f, g, s: annotation for each of the taxa ranks, namely kingdom/domain, phylum, class, order, family, genus, and species

The *xls file is an Excel file combining data from the *.tab and ".anno files. Using biom.pl, a ".biom file can be subsequently used by QIIME [19]. We have implemented options for generating annotations to either SILVA [24] or Greengenes [21]. All 4 files types can automatically be generated if RiboTagger is run in batch mode.

\section{Conclusions}

Here, we have developed software for the fast recovery of SSU-RNA sequences from shotgun nucleic acid surveys of complex microbial communities. Our code is fast, completing an analysis of about $40 \mathrm{M}$ reads within $1.5 \mathrm{~h}$, and will output an annotated matrix of read counts that can be used for downstream community profiling analysis with minimal further processing. Our implementation executes in a single line, avoiding the complications and the lack of robustness inherent in combination-type pipelines and is at least 6 times faster than SSUsearch [17] and RiboFrame [16]. Additional, we also note that our approach avoids the use of OTU generation, which recent analyses suggest may carry significant advantages in resolving intra-community dynamics for some classes of experimental design, such as time series experiments [25].

\section{Availability and requirements}

Project name: RiboTagger

Project home page: https://github.com/xiechaos/ribotag ger

Operating system (s): Platform independent

Programming language: Perl

Other requirements: None

License: GPLv3

Any restrictions to use by non-academics: none.

\section{Abbreviations}

OTU: Operational taxonomic unit; PSSM: Position specific scoring matrix; RS: Recognition sequence; TS: Tag sequence

\section{Acknowledgements \\ We thank our colleagues Larry Liew, Stefan Wuertz, Yehuda Cohen, Uma Shankari d/o Chanda Segaran, Stephan C. Schuster, Daniela Moses for providing access to data, and Siao Yun Chang, Choon Leng Lau and their colleagues from the Public Utilities Board (Republic of Singapore) for assistance with access to facilities and sampling operations.}

\section{Declarations}

This article has been published as part of BMC Bioinformatics Volume 17 Supplement 19, 2016. 15th International Conference On Bioinformatics (INCOB 2016): bioinformatics. The full contents of the supplement are available online https://bmcbioinformatics.biomedcentral.com/articles/ supplements/volume-17-supplement-19

\section{Funding}

Publication charges for this article were supported in full by the Singapore National Research Foundation and the Ministry of Education under the Research Centre of Excellence Programme.

\section{Availability of data and material}

The sequencing datasets analyzed during the current study are not yet publicly available due to their forming part of a large set of data being released as part of a primary manuscript. They are available on request to R.B.H.W.

\section{Authors' contributions}

C.X. conceived and designed the approach and implemented methods. C.X., C. L. W.G and R.B.H.W analyzed example data, and all authors interpreted the results. The paper was written by C.X, D.H.H and R.B.H.W with specific contributions from C.L.W.G and P.F.R.L. All authors read and approved the final manuscript.

\section{Competing interests}

The authors declare that they have no competing interests.

Ethics approval and consent to participate

Not applicable.

\section{Consent for publication}

Not applicable.

\begin{abstract}
Author details
${ }^{1}$ Singapore Centre for Environmental Life Sciences Engineering, National University of Singapore, Singapore 117456 , Singapore. ${ }^{2}$ Centre for Bioinformatics, Tuebingen University, Tuebingen 72076, Germany. ${ }^{3}$ Life Sciences Institute, National University of Singapore, Singapore 117456, Singapore. ${ }^{4}$ Department of Biochemistry, Yong Loo Lin School of Medicine, National University of Singapore, Singapore 117596, Singapore. ${ }^{5}$ Current address: Human Longevity Inc, Singapore, Singapore.
\end{abstract}

Published: 22 December 2016

\section{References}

1. Raes J, Bork P. Molecular eco-systems biology: towards an understanding of community function. Nat Rev Microbiol. 2008;6:693-9. 
2. Harismendy $\mathrm{O}$, et al. Evaluation of next generation sequencing platforms for population targeted sequencing studies. Genome Biol. 2009;10:R32.

3. Liu Z, Lozupone C, Hamady M, Bushman FD, Knight R. Short pyrosequencing reads suffice for accurate microbial community analysis. Nucleic Acids Res. 2007;35:e120.

4. Haas BJ, et al. Chimeric $16 \mathrm{~S}$ rRNA sequence formation and detection in Sanger and 454-pyrosequenced PCR amplicons. Genome Res. 2011;21:494-504.

5. Hong S, Bunge J, Leslin C, Jeon S, Epstein SS. Polymerase chain reaction primers miss half of rRNA microbial diversity. ISME J. 2009:3:1365-73.

6. Albertsen M, Karst SM, Ziegler AS, Kirkegaard RH, Nielsen PH. Back to BasicsThe Influence of DNA Extraction and Primer Choice on Phylogenetic Analysis of Activated Sludge Communities. PLoS ONE. 2015;10, e0132783.

7. Thomas T, Gilbert J, Meyer F. Metagenomics-a guide from sampling to data analysis. Microb Inform Exp. 2012;2:3.

8. Albertsen $\mathrm{M}$, et al. Genome sequences of rare, uncultured bacteria obtained by differential coverage binning of multiple metagenomes. Nat Biotechnol. 2013;31:533-8.

9. Chistoserdova L. Is metagenomics resolving identification of functions in microbial communities? Microb Biotechnol. 2014;7:1-4.

10. Hartmann M, Howes CG, Abarenkov K, Mohn WW, Nilsson RH. V-Xtractor: an open-source, high-throughput software tool to identify and extract hypervariable regions of small subunit (16S/18S) ribosomal RNA gene sequences. J Microbiol Methods. 2010;83:250-3.

11. Lee JH, Yi H, Chun J. rRNASelector: a computer program for selecting ribosomal RNA encoding sequences from metagenomic and metatranscriptomic shotgun libraries. J Microbiol. 2011:49:689-91.

12. Bengtsson J, et al. Metaxa: a software tool for automated detection and discrimination among ribosomal small subunit (12S/16S/18S) sequences of archaea, bacteria, eukaryotes, mitochondria, and chloroplasts in metagenomes and environmental sequencing datasets. Antonie Van Leeuwenhoek. 2011;100:471-5.

13. Bengtsson-Palme J, et al. METAXA2: improved identification and taxonomic classification of small and large subunit rRNA in metagenomic data. Mol Ecol Resour. 2015;15:1403-14.

14. Leach AL, Chong JP, Redeker KR. SSuMMo: rapid analysis, comparison and visualization of microbial communities. Bioinformatics. 2012;28(5):679-86.

15. Logares $\mathrm{R}$, et al. Metagenomic $16 \mathrm{~S}$ rDNA Illumina tags are a powerful alternative to amplicon sequencing to explore diversity and structure of microbial communities. Environ Microbiol. 2014;16:2659-71.

16. Ramazzotti M, Berná L, Donati C, Cavalieri D. riboFrame: An Improved Method for Microbial Taxonomy Profiling from Non-Targeted Metagenomics. Front Genet. 2015;6:329.

17. Guo J, Cole JR, Zhang Q, Brown CT, Tiedje JM. Microbial Community Analysis with Ribosomal Gene Fragments from Shotgun Metagenomes. Appl Environ Microbiol. 2015;82(1):157-66.

18. Caporaso JG, et al. QIIME allows analysis of high-throughput community sequencing data. Nat Methods. 2010;7:335-6.

19. Huson DH, et al. MEGAN Community Edition-Interactive Exploration and Analysis of Large-Scale Microbiome Sequencing Data. PLoS Comput Biol. 2016:12, e1004957.

20. Cole JR, et al. Ribosomal Database Project: data and tools for high throughput rRNA analysis. Nucleic Acids Res. 2014;42(Database issue):D633-42.

21. Pruesse E, et al. SILVA: a comprehensive online resource for quality checked and aligned ribosomal RNA sequence data compatible with ARB. Nucleic Acids Res. 2007;35:7188-96.

22. Giovannoni SJ, et al. Genome Streamlining in a Cosmopolitan Oceanic Bacterium. Science. 2005;309:1242-5.

23. Tikhonov M, Leach RW, Wingreen NS. Interpreting 165 metagenomic data without clustering to achieve sub-OTU resolution. ISME J. 2015;9:68-80.

24. DeSantis TZ, et al. Greengenes, a chimera-checked 16S rRNA gene database and workbench compatible with ARB. Appl Environ Microbiol. 2006;72: 5069-72.

\section{Submit your next manuscript to BioMed Central and we will help you at every step:}

- We accept pre-submission inquiries

- Our selector tool helps you to find the most relevant journal

- We provide round the clock customer support

- Convenient online submission

- Thorough peer review

- Inclusion in PubMed and all major indexing services

- Maximum visibility for your research

Submit your manuscript at www.biomedcentral.com/submit

) Biomed Central 